\section{THE USABILITY OF PHYSICAL ACTIVITY AND COGNITIVE TRAINING APPLICATIONS IN PEOPLE WITH MILD COGNITIVE IMPAIRMENT}

\section{Smith, University of Alabama in Huntsville}

Evidence has shown that exercise, a healthier diet, and smoking cessation may protect the brain, but evidence is scarce on exercise combined with cognitive training and its benefits for people with mild cognitive impairment. The aim of this study is to identify key issues with utility, effectiveness, and appeal of specific electronic applications for people with mild cognitive impairment in order to guide the design and development of a mobile application which incorporates both physical and cognitive activities, which may improve, impede or prevent cognitive decline. Sixteen participants, 65 and older, with mild cognitive impairment, assessed using the Montreal Cognitive Assessment tool, with a range of 19-25 were recruited. To assess the participants' ability to consent to the study, the MacArthur Competence Assessment Tool for Clinical Research was used. Participants were observed playing a physical activity application and cognitive training application via a tablet, on two separate occasions. A Usability Observation form was used to obtain data on facial features as well as verbal and body language while playing. A survey and focus group sessions were held to get feedback from the participants. The majority of the participants were able to use the tablets and play the physical activity and cognitive training applications. However, some of the applications had levels that were more difficult for some of the participants, but a few said parts of the applications were too easy. The investigators noted that a 'tablet stand' would have highly enhanced participation during the use of physical activity applications.

\section{USE OF MOBILE TECHNOLOGY TO ASSESS BALANCE IN OLDER ADULTS WITH AND WITHOUT SYMPTOMS DURING A SIT TO STAND MANUEVER \\ D. Gray-Miceli ${ }^{1}$, W. Craelius ${ }^{2}$, N. Patel ${ }^{3}$, J. Galvin ${ }^{1}, 1$. Florida Atlantic University, 2. Rutgers University School of Biomedical Engineering, 3. Rutgers University, School of Biomedical engineering}

Gaps in science exist to prevent treatable falls, such as those due to orthostatic hypotension, because of a myriad of issues related to its detection. Real-time motion data during activities such as the Sit to Stand $(\mathrm{StS})$ maneuver, which can produce $\mathrm{OH}$, are not captured by fall risk tools. And while Smartphones have been used to assess balance, sensing technology has not integrated the person's symptoms with real time motion data. In our pilot using a Smartphone with tri-axial accelerometer and gyroscope sensors, motion data was generated and acceleration values were collected over a 30 -second time period during the StS maneuver in 23 elderly residents of a 100 bed subacute rehabilitation unit in a nursing home. The real number acceleration values were squared in the $\mathrm{X}, \mathrm{Y}$ and $\mathrm{Z}$ directions and summed together over each ten-second time interval. This summation of the $\mathrm{X}, \mathrm{Y}$, and $\mathrm{Z}$ direction squared acceleration values each represent a coefficient of excessive sway over the time interval. Six coefficients of excessive sway, three for each participant at three points in time were produced. Coefficients for each time interval were summed to produce a cumulative coefficient. Findings from four exemplars are presented which describe the clinical correlation of subjective and objective findings related to $\mathrm{OH}$, lightheadedness, mean arterial and pulse pressure changes. Symptomatic $\mathrm{OH}$ was associated with the most dramatic change in pressures and highest coefficients of sway. Technology which integrates patient symptoms with objective findings is critical to advance the science in injurious falls prevention.

\section{VALIDITY OF SENSOR-BASED, HABITUAL PHYSICAL ACTIVITY AND GAIT ANALYSIS IN MULTIMORBID, OLDER PERSONS}

K. Hauer ${ }^{1}$, M. Bongartz ${ }^{2}$, R. Kiss ${ }^{2}$, A. Lacroix ${ }^{2}$, P. Ullrich ${ }^{3}$, T. Eckert ${ }^{4}$, C. Jansen ${ }^{5}$, S. Mellone ${ }^{6}$, 1. Bethanien-Hospital /Geriatric Centre at the University of Heidelberg, 2. Agaplesion Bethanien Hospital, Heidelberg, BadenWürttemberg, Germany, 3. Bethanien Hospital Geriatric Center at the University of Heidelberg, 4. Agaplesion Bethanien Hospital Heidelberg, 5. Network of ageing Research (NAR) at the University of Heidelberg, 6. Department of Electrical, Electronic, and Information Engineering, University of Bologna

The aim of the study was to investigate the biometrical quality of a newly developed activity monitor (uSense) to document established physical activity (PA) parameters as well as innovative qualitative and quantitative gait characteristics for habitual activity behavior in multi-morbid, older adults. Validity, testretest reliability, and feasibility of established (including activity counts, MET-Intensities, number/duration of gait episodes/ steps) as well as newly developed gait characteristics, which have not been documented before for habitual assessment (including number, velocity, duration of turnings, various parameters for gait symmetry/ regularity), have been analyzed for multimorbid, geriatric patients with cognitive impairment $(n=110)$ discharged from ward-based rehabilitation. On average, Spearman correlations of established and innovative uSense parameters with clinically relevant parameters were high for motor performances (range for rhos: $0.02-0.63$ ) and life space $(0.01-0.59)$ and low to moderate for cognitive status $(0.01-0.25)$, and age $(0.01-$ 0.30 ), indicating moderate to good construct validity. Concurrent validity was high as PA parameters measured by the U-Sense monitor showed consistently high correlation with equivalent parameters measured by another well-established ambulatory motion sensor (PAMSysTM) $(0.59$ - 0.91). Moderate to excellent test-retest reliability was shown for all uSense parameters (ICC: $0.68-0.97$ ) and good feasibility could be shown, as $85.5 \%$ of all measurements were completed without failure. The uSense monitor allows documentation of established and innovative qualitative-quantitative parameters for habitual PA behavior which have so far only been assessed in laboratory settings in multi-morbid, cognitively impaired, older adults with moderate to good validity and high test-retest reliability.

\section{SESSION 2295 (POSTER)}

\section{CIVIC ENGAGEMENT, SOCIALIZATION, AND WELL-BEING}

\section{ACTIVITY ENGAGEMENT AND COGNITION: FINDINGS FROM A COMMUNITY-DWELLING U.S. CHINESE AGING POPULATION STUDY}

F. Tang ${ }^{1}$, I. Chi ${ }^{2}$, W. Zhang ${ }^{3}$, X. Dong ${ }^{4}, 1$. University of Pittsburgh, 2. University of Southern California, 3. 